\title{
Unfolding measurement of the Atmospheric Muon Neutrino Spectrum using IceCube-79/86
}

\section{The IceCube Collaboration ${ }^{\dagger}$,}

${ }^{\dagger}$ http://icecube.wisc.edu/collaboration/authors/icrc15_icecube

E-mail: mathis.boerneraicecube.wisc.edu

IceCube is a cubic kilometer neutrino telescope located at the geographic South Pole. Although primarily designed for the detection of cosmic neutrinos, the detector is well suited for measurements of the atmospheric muon neutrino energy spectrum. We present the measurement of this spectrum obtained in its 79 and its full 86-string configuration. The analysis was carried out using dedicated machine learning algorithms for the selection of neutrino candidates. The spectrum was obtained by applying regularized unfolding as implemented in the unfolding software TRUEE. An additional component due to a diffuse flux of high energy astrophysical neutrinos has been observed in addition to the flux of conventional atmospheric neutrinos.

Corresponding authors: M. Börner ${ }^{1 *}$, T. Ruhe ${ }^{1}$, F. Scheriau ${ }^{1}$, M. Schmitz ${ }^{1}$

${ }^{1}$ Dept. of Physics, Technical University of Dortmund, 44227 Dortmund, Germany

The 34th International Cosmic Ray Conference,

30 July - 6 August, 2015

The Hague, The Netherlands

\footnotetext{
*Speaker.
} 


\section{Introduction}

IceCube is a neutrino observatory with an instrumented volume of one cubic kilometer [1]. The detector consists of 5160 digital optical modules (DOMs). The DOMs are attached to 86 strings. The strings are deployed at depths between $1450 \mathrm{~m}$ and $2450 \mathrm{~m}$ in glacial ice at the geographical south pole. The purpose of the DOMs is to measure Cherenkov light emitted by charged particles propagating through the detector. In this analysis muon neutrinos are searched for with throughgoing muons produced in charged current neutrino interactions.

The unfolded spectra cover an $\mathrm{E}_{V}$-range of more than four orders of magnitude from $250 \mathrm{GeV}$ up to $3.2 \mathrm{PeV}$. Up to $\mathrm{TeV}$ energies the muon neutrino spectrum is expected to be dominated by atmospheric neutrinos produced in decays of pions and kaons in cosmic ray air showers. This component is called conventional. At higher energies additional neutrinos from the decay of shortlived charmed mesons start to contribute significantly to the spectrum. This component is called prompt. Both are purely atmospheric. In 2013 IceCube was the first experiment to prove the existence of a third component, the astrophysical component [2]. Those neutrinos are expected to come from active galactic nuclei or gamma ray bursts, but the exact sources are not known yet.

\section{Data sample and Analysis}

The first year of data analyzed was taken in IceCube's 79-string configuration (IC79) between May 2010 and May 2011. The second year was the first year of IceCube's 86-string configuration (IC86) and data was taken between May 2011 and May 2012. To separate cosmic ray-induced muons from neutrino-induced muons, the earth is used for shielding against atmospheric muons in this analysis. Hence, the datasets only cover the northern hemisphere. For each year separate processes using machine learning algorithms were applied to obtain highly pure neutrino datasets. The core of the separation is a Random Forest [3]. To ensure a good performance for the Random Forest, it is preceded by a maximum relevance minimum redundancy variable selection $[4,5]$. The approach is based on the process described in [6]. The separation leads to 66885 muon neutrino candidates with an estimated purity of $(99.5 \pm 0.3) \%$ for the first year and 92519 muon neutrino candidates with an estimated purity of $(99.6 \pm 0.2) \%$ for the second year.

The neutrino energy cannot be measured directly. Thus, the spectrum has to be reconstructed from measurable variables correlated to the neutrino energy. One possibility to solve this inverse problem is unfolding. For this analysis both datasets were unfolded separately with a regularized unfolding method implemented in the software TRUEE [7].

\section{Energy Spectra for the Northern Hemisphere}

The zenith range of the datasets spans from $86^{\circ}$ ( $4^{\circ}$ above the horizon) to $180^{\circ}$ (north pole). The unfolded spectra in comparison to theoretical predictions are depicted in Fig. 1. While the spectrum of the year before IC79 (IC59) [6] showed no hints of an astrophysical component, both the IC79 and the IC86 spectrum start to exceed an atmospheric only prediction around $E_{v} \approx 50 \mathrm{TeV}$. The excess of the IC79 spectrum is significantly higher and not compatible with an atmospheric only prediction within its errors. 


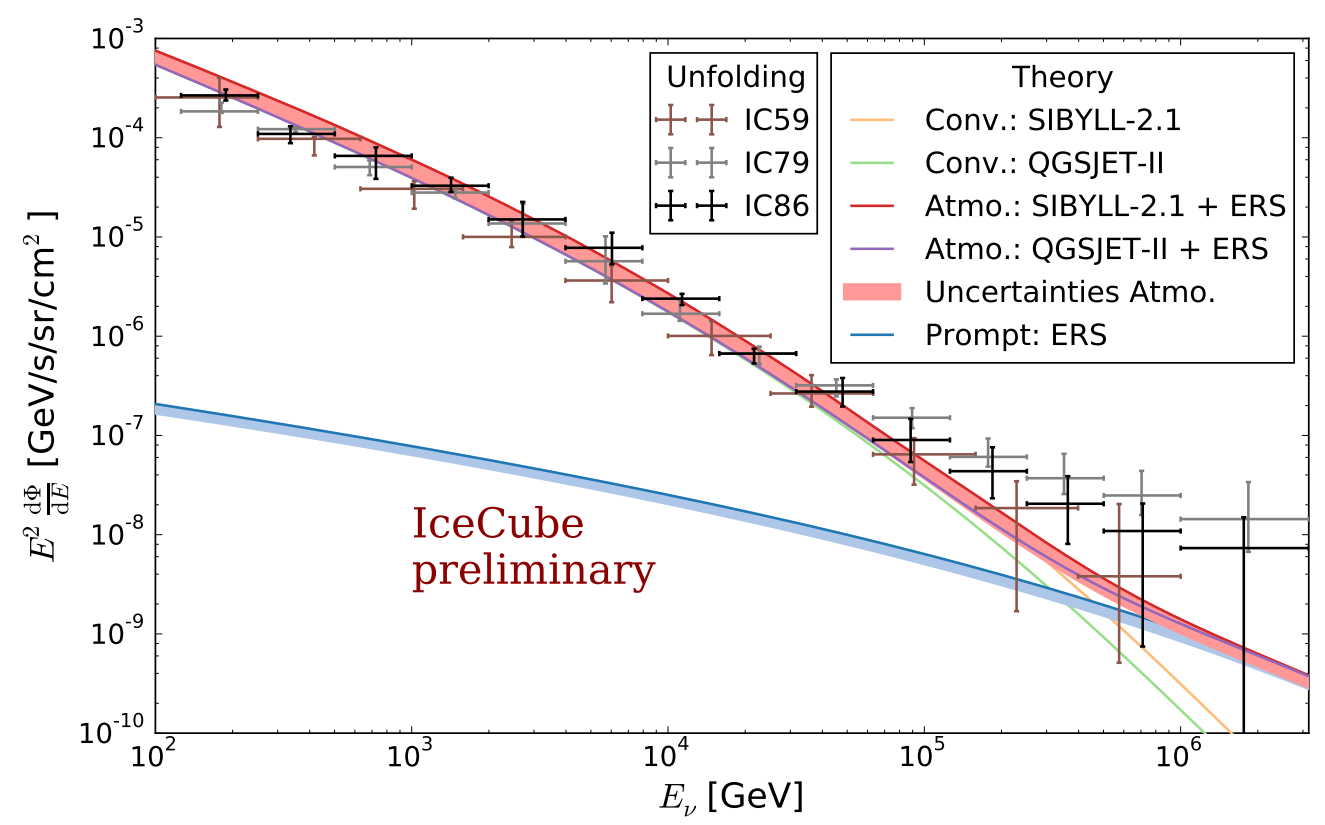

Figure 1: The unfolded spectra for IC79 and IC86 are shown in gray and black. The unfolded spectrum for the year before IC79 (IC59) is shown in brown [6]. For comparison, theoretical predictions for the conventional component QGSJET-II [8] (green) as a lower bound and SYBILL-2.1 [8] (yellow) as a upper bound are shown. Most of the common predictions, like those .Honda et al. [9], are in between those bounds. For the prompt component the prediction by Enberg et al. [10], with the bounds also given in the paper, is shown. In addition the sum of the conventional and the prompt flux is shown for both conventional models. All theoretical predictions are calculated for the primary spectrum by Gaisser [11].

Because the methods and the energy binning for the IC79 and IC86 spectrum are the same, it is reasonable to calculate the weighted average for both spectra to obtain a combined IC79 and IC86 spectrum. This averaged spectrum in comparison to recent IceCube measurements and theoretical prediction for the atmospheric flux is depicted in Fig. 2. The comparison shows that the observed excess is compatible with the recent measurements of the astrophysical component.

\section{Zenith Dependent Unfolding}

The unfolded spectrum is a measurement of the sum of the conventional, prompt and astrophysical component. It is not possible to disentangle the astrophysical from the atmospheric component without knowing the shape and the normalization of the conventional and prompt flux.

Pions and kaons producing conventional muon neutrinos have a shorter interaction than decay length. Therefore, they interact in the atmosphere resulting in a loss of energy and steepening of the muon neutrino energy spectrum. The length of the path through the atmosphere and therefore the amount of energy lost before the decay depends on the zenith angle. The prompt muon neutrinos on the other hand originate from decays of short-lived mesons decaying directly after production. Therefore, the prompt neutrino flux has no additional zenith dependency. All components have a common zenith dependence due to the increase of the neutrino cross sections for high energies. 


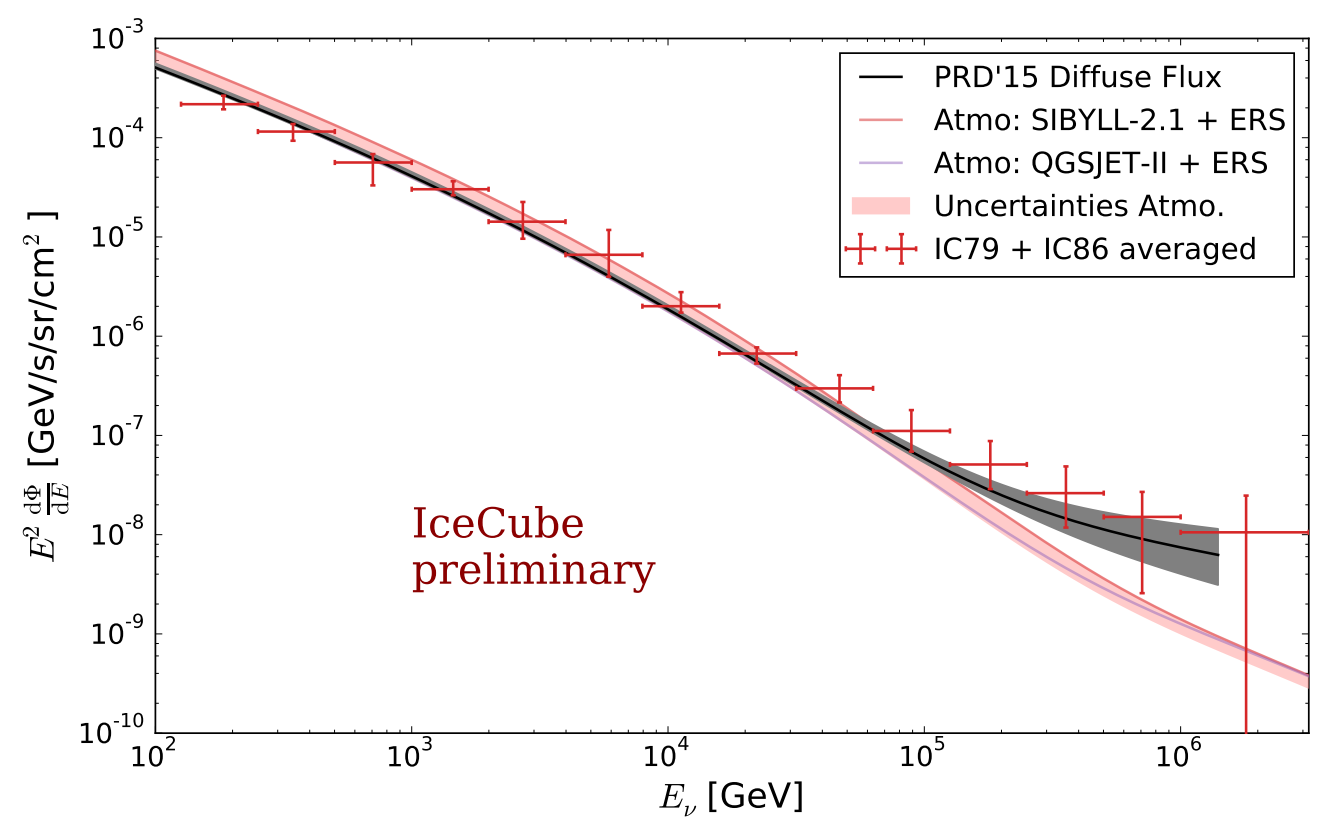

Figure 2: The combined IC79 and IC86 spectrum is shown in red. The spectra were combined with a weighted average. The theoretical predictions for the atmospheric flux are shown as in Fig. 1. The best fit from an IceCube analysis of high-energy neutrinos that interacted inside the detector volume during the same two data taking years [12] is shown in black. The best fit contains $0.97_{-0.03}^{+0.10}$ times the conventional prediction by Honda et al. [9] and $0.00_{-0.00}^{+1.57}$ times the prompt prediction by Enberg et al. [10]. Both fluxes are calculated for the primary spectrum by Gaisser [11]. The additional astrophysical component is described with an unbroken power law $\left(2.06_{-0.26}^{+0.35}\right) \cdot 10^{-18} \cdot(E / 100 \mathrm{TeV})^{-2.46 \pm 0.12} \mathrm{GeV}^{-1} \mathrm{~s}^{-1} \mathrm{sr}^{-1} \mathrm{~cm}^{-2}$.

This results in an increasing opaqueness of the earth core and a survival probability $<1 \%$ for neutrinos at $E_{v} \approx 1 \mathrm{PeV}$ when propagating through the earth core [13].

For the IC86 dataset the statistics are large enough to unfold the dataset in three different zenith bands. The resulting spectra are shown in Fig. 3. Despite IC86 being the largest neutrino dataset unfolded so far, the decreasing statistics in each band especially at the high energies is a big challenge for the zenith dependent unfolding.

The upper energy reach is limited at $E \approx 125 \mathrm{TeV}$ for the band looking through the earth and $E_{v} \approx 250 \mathrm{TeV}$ for the other two bands. The unfolded spectra are compatible with theoretical predictions within their errors. For more insight in the composition more zenith bands with smaller errors are needed.

\section{Conclusion}

The unfolding of the muon neutrino energy spectrum for IC79 and IC86 extend the upper energy limit by a factor of $\sim 2.5$ to $3.2 \mathrm{PeV}$ in comparison to previous measurements by IceCube with data from the 59-string configuration [6]. The resulting spectra measure an excess over the atmospheric prediction at the highest energies, showing the transition from a spectrum dominated by the conventional flux to a harder spectrum. The measured excess is compatible with recent 


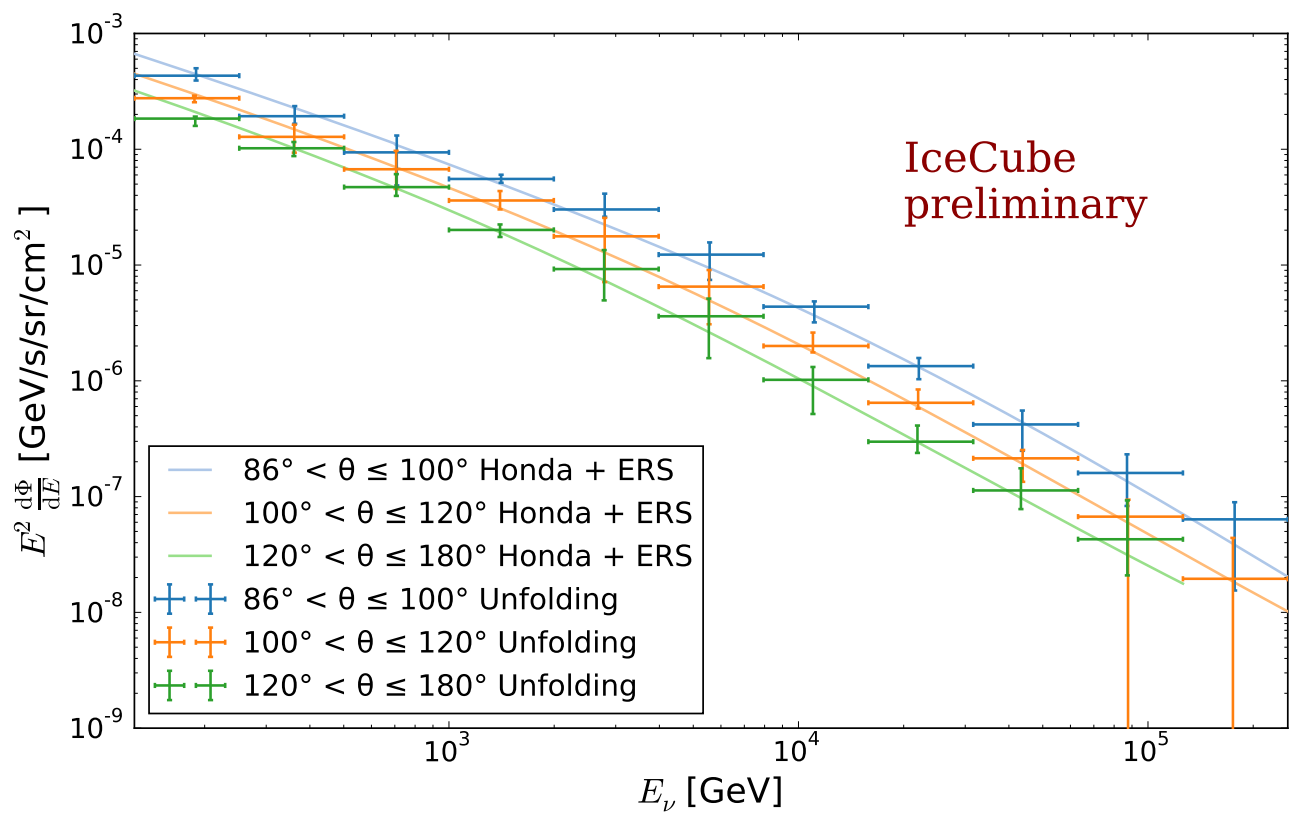

Figure 3: Unfolded energy spectra for different zenith bands for IC86 data. The shown theoretical curves are the sum of conventional flux by Honda et al. [9] and prompt flux by Enberg et al. [10] calculated with the primary spectrum by Gaisser [11].

measurements of the astrophysical neutrino flux in IceCube [12,14]. Although the unfolding cannot quantify a significance for the existence of an astrophysical component, these measurements strengthen the evidence for an additional, non-atmospheric flux from the northern hemisphere.

The unfolding measures the sum of the atmospheric and the astrophysical flux. To disentangle the astrophysical component, the shape and normalization of the conventional and the prompt flux has to be known precisely. To get deeper insight in the atmospheric flux composition, an unfolding in three zenith bands was performed. The resulting spectra are compatible with the atmospheric prediction by Honda et al. [9] and Enberg et al. [10]. For a better determination of the atmospheric components an unfolding in more zenith bands is needed.

\section{References}

[1] IceCube Collaboration, A. Achterberg et al., Astroparticle Physics 26 (2006), no. 3 $155-173$.

[2] IceCube Collaboration, I. Collaboration et al., Science 342 (2013), no. 61611242856.

[3] L. Breiman, Machine learning 45 (2001), no. 1 5-32.

[4] H. Peng, F. Long, and C. Ding, Pattern Analysis and Machine Intelligence, IEEE Transactions on 27 (2005), no. 8 1226-1238.

[5] M. A. Hall, Correlation-based feature selection for machine learning. PhD thesis, The University of Waikato, 1999. 
[6] IceCube Collaboration, M. G. Aartsen et al., The European Physical Journal C 75 (2015), no. $31-14$.

[7] N. Milke, M. Doert, S. Klepser, D. Mazin, V. Blobel, and W. Rhode, Nuclear Instruments and Methods in Physics Research Section A: Accelerators, Spectrometers, Detectors and Associated Equipment 697 (2013) 133-147.

[8] A. Fedynitch, J. B. Tjus, and P. Desiati, Physical Review D 86 (2012), no. 11114024.

[9] M. Honda, T. Kajita, K. Kasahara, S. Midorikawa, and T. Sanuki, Physical Review D 75 (2007), no. 4043006.

[10] R. Enberg, M. H. Reno, and I. Sarcevic, Physical Review D 78 (2008), no. 4043005.

[11] T. K. Gaisser, Astroparticle Physics 35 (2012), no. 12 801-806.

[12] IceCube Collaboration, M. G. Aartsen et al., Physical Review D 91 (2015), no. 2022001.

[13] I. F. Albuquerque, J. Lamoureux, and G. F. Smoot, The Astrophysical Journal Supplement Series 141 (2002), no. 1195.

[14] IceCube Collaboration, M. G. Aartsen et al., Physical review letters 113 (2014), no. 10 101101. 